\title{
SÍNTOMAS DE DEPRESIÓN EN MUJERES SOLTERAS CON JEFATURA DE FAMILIA EN PUERTO RICO: IMPLICACIONES PARA LAS PROFESIONES DE AYUDA
}

Dra. Nilsa M. Burgos Ortiz'

Dra. Milagros Bravo

Dra. Mary Clare Lennon

\section{Introducción}

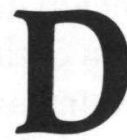

iversas investigaciones en salud mental han demostrado consistentemente que las mujeres tienen tasas más altas de depresión, al igual que de síntomas de depresión, que los hombres (Myers et al., 1984; Robin et al., 1984; Weissman y Klerman, 1978). Este hallazgo ha sido confirmado tanto en poblaciones hispanas de los Estados Unidos (Vega et al., 1987; Vernon y Roberts, 1982) como en Puerto Rico (Canino et al., 1987a). Aún más, las mujeres solteras con jefatura de familia, en comparación con otras mujeres, demuestran un riesgo más alto para los síntomas psiquiátricos, particularmente síntomas de depresión, (Belle, 1980; Bennett, 1987; McLanahan, 1985). Sin embargo, este último hallazgo no ha sido estudiado con mujeres puertorriqueñas.

\footnotetext{
1 - Dra. Nilsa M. Burgos Ortiz, Catedrática, Escuela Graduada de Trabajo Social, Universidad de Puerto Rico.

- Dra. Milagros Bravo, Catedrática Asociada, Estudios Graduados en Educación e Instituto de Investigaciones en Ciencias de la Conducta, Universidad de Puerto Rico.

- Dra. Mary Clare Lennon, Catedrática Auxiliar, Escuela de Salud Pública, Columbia University.
} 
El presente estudio examina este asunto para las mujeres en Puerto Rico, utilizando un banco de datos de la población adulta de Puerto Rico. En el estudio se consideran mujeres solteras con jefatura de familia aquellas mujeres divorciadas, separadas, viudas o solteras que asumen la responsabilidad económica principal de sus hijos, hijas y otros miembros de su familia y que no tienen esposo o compañero permanente. El estudio tiene también el propósito de identificar factores sociales, como responsabilidades familiares, eventos de vida y redes de apoyo deficientes, que pueden contribuir al riesgo de síntomas de depresión en estas mujeres.

El Censo de la Población de 1990 señala que el 18 por ciento de los hogares hispanos en Estados Unidos tenían una mujer en su jefatura, comparado con el 12 por ciento de los hogares que no eran hispanos (US Bureau of the Census, 1991a). En ese mismo año, la tasa fue aún más alta en Puerto Rico: 23 por ciento de los hogares tenían una mujer en su jefatura (203,508 hogares), un aumento del 14 por ciento $(88,164$ hogares) que existía en 1970 (US Bureau of the Census, 1991b y 1972). Las mujeres jefes de familia, generalmente confrontan más dificultades que otras mujeres. Por ejemplo, tienden a vivir en mayor número en condiciones de pobreza (Bennett, 1987; Kamerman, 1988): en 1990 , un 70 por ciento de las familias con una mujer en su jefatura estaban por debajo del nivel de pobreza (U.S. Bureau of the Census, 1991b). Sin embargo, la pobreza no es el único problema para estas mujeres, sino también el prejuicio, bajos niveles de educación, responsabilidad por niños(as) dependientes, menos destrezas ocupacionales y mayor aislamiento. Todas estas situaciones las coloca en riesgo de sufrir depresión y estrés (Belle, 1980; Bennett, 1987; Colberg y Burgos, 1988; Costin, 1987; Mclanahan, 1985; Morrison, et al., 1986; Vega, et al., 1986).

A pesar del aumento de mujeres solteras hispanas con jefatura de familia y de sus múltiples problemas, los estudios de esta población son muy escasos. Los resultados del presente estudio pueden ampliar el entendimiento del bienestar psicológico de las mujeres hispanas. A pesar de que cada grupo hispano es único, no obstante comparten experiencias culturales comunes, como el uso del lenguaje y los roles de género. Dada las similitudes culturales, mucho de lo que se entienda acerca de la mujer puertorriqueña puede ser pertinente a 
otras mujeres hispanas.

El estudio que aquí informamos está conceptualmente enmarcado en las teorías de género y estrés, al igual que en estudios previos, basados principalmente en muestras de mujeres blancas en Estados Unidos. Los altos niveles de depresión o síntomas de depresión en mujeres solteras con jefaturas de familia se deben probablemente a la forma en que se construyen los géneros socialmente. Mientras que el rol del hombre ha sido definido como instrumental, dándole énfasis a la independencia, competencia y racionalidad, el rol de la mujer se concibe como expresivo, con énfasis en la ternura, la compasión y el apoyo (Bem, 1974). Aún más, esta visión postula que los hombres contribuyen a la sociedad en la esfera pública mientras que la contribución de la mujer se limita a los quehaceres domésticos en la esfera privada (Lipman-Blumen, 1984). Estas diferencias de género, a pesar de ser prevalentes en la mayoría de las sociedades, son especialmente evidentes en la cultura hispana.

A pesar de los cambios en la cultura puertorriqueña debidos al desarrollo socio-económico y la exposición a la cultura estadounidense, estos rasgos culturales tradicionales aún están vigentes en muchos contextos en la Isla. Los roles de género tradicionales aún prescriben las responsabilidades familiares como lo más importante en la vida de las mujeres. Es probable que mucho del estrés sentido por las mujeres solteras con jefatura de familia se derive de estos roles. Mujeres con muchos hijos(as) o con menores de seis años de edad han sido señaladas como particularmente vulnerables a la depresión, cuando se compara con otras mujeres (Bennett, 1987); Brown, 1978). Aún más, las madres puertorriqueñas usualmente se preocupan por sus hijos(as) aún cuando no vivan con ella. Las mujeres, también son responsables de cuidar a sus padres y madres envejecientes. En un estudio de viudas sobre 60 años de edad, Sánchez (1984), encontró que las hijas eran su principal fuente de apoyo.

Además de estas responsabilidades familiares, que la mayor parte de las mujeres comparte, las mujeres solteras con jefatura de familia pueden confrontar dificultades adicionales. En Puerto Rico, como en otras sociedades tradicionales, se supone que una mujer con hijos(as) tenga un hombre a su lado para su protección y sustento 
económico, al igual que para disciplinar a los hijos(as) (Burgos, 1986). No obstante, las mujeres solteras con jefatura de familia tienen que asumir usualmente roles económicos y disciplinarios, a la vez que ofrecer apoyo y afecto, teniendo así que ejercer funciones instrumentales y expresivas simultáneamente. Debido a que estos roles son definidos culturalmente como mutuamente exclusivos, más que como capaces de ser integrados, estas mujeres tienden a sentir estrés al lidiar con demandas conflictivas e incompatibles.

Se espera que estas mujeres experimenten aún más estrés si, además de sus responsabilidades como jefas de familia, ellas tienen que lidiar con eventos de vida desorganizadores como son el desempleo y la muerte de un ser querido. Los eventos que involucran circunstancias nuevas, intensas, inesperadas y de rápido cambio han sido identificados como fuentes de estrés (Zegans, 1982). La convergencia de cambios desorganizadores en la vida en un período limitado de tiempo tiende a producir problemas psicológicos (Holmes y Rahe, 1967). Debido a que las mujeres solteras con jefatura de familia no tienen un compañero estable con quien compartir las demandas que estos cambios desorganizadores imponen, ellas podrían estar más propensas a exhibir sintomatología psiquiátrica.

Para explicar las formas en las cuales los estresores pueden afectar el bienestar humano, Dohrenwend y Dohrenwend (1981) desarrollaron varios modelos conceptuales. El modelo de vulnerabilidad plantea que la gente con ciertas características socio-demográficas (como serían las mujeres solteras con jefatura de familia) cuando son confrontadas con estresores específicos mostrarán más estrés que grupos comparables. En contraste, el modelo aditivo establece que las características socio-demográficas y los estresores se relacionan independientemente y aditivamente a la sintomatología. Existe evidencia empírica que provee apoyo al modelo de vulnerabilidad entre ciertos grupos. Por ejemplo, se ha encontrado que personas que no se han casado están en mayor riesgo de experimentar estrés psicológico después de un evento estresante (Ver Thoits, 1987, para una discusión de esta literatura). En un estudio en el que se utiliza el mismo banco de datos que en la presente investigación (Solomon, et al., 1993), gente soltera sin familia (ni cónyuge ni hijos o hijas) exhibieron más síntomas psicológicos después de la exposición a un desastre. 
Contrario a lo que se esperaba, no sucedió así con padres o madres solteras. Sin embargo, el estudio concentró en ambos géneros, sin considerar jefatura de familia y en un evento extraordinario, no en eventos de vida comunes. Si las mujeres solteras con jefatura de familia son más vulnerables que otras mujeres a eventos de vida estresantes está aún sin investigar.

El apoyo social ha sido identificado como un componente importante en la relación estrés y disfunción psicológica (Ver Cohen y Syme, 1985, para una revisión de esta literatura). El concepto de apoyo social ha sido definido en diferentes formas pero definiciones usualmente incluyen relaciones humanas en la cual se provea cooperación y ayuda de naturaleza emocional (afecto, amor y empatía) o instrumental (bienes y servicios) (Vea por ejemplo, House, 1981). Varias de las dimensiones del concepto han sido conceptualizadas e identificadas empíricamente (Barrera, 1986; Tardy, 1985) como estructura (por ejemplo, tamaño de la red de apoyo o número de personas que proveen apoyo) y calidad (por ejemplo, satisfacción con el apoyo). Generalmente, la evidencia empírica ha documentado que el apoyo tiene una influencia positiva en la salud y bienestar tanto por sí mismo o como mediadora de estrés (Cohen y Syme, 1985).

Algunos estudios han encontrado que el apoyo social funciona como protector para las madres solteras que confrontan estrés (D'Ercole, 1988). Se ha encontrado que las redes de apoyo social tienden a reducir los problemas y el sentido de aislamiento en madres solteras de bajos ingresos (Wells y Ray, 1986). No obstante, las madres solteras señalan tener menos personas en sus redes de apoyo (Naran, 1989) y estar menos satisfechas cuando sus redes de apoyo están compuestas por familiares (D'Ercole, 1988). De igual forma, Solomon y sus colegas (1993) encontraron que a los padres solteros que estuvieron expuestos al desastre, comparados con los padres solteros que no lo estuvieron, se les habían reducido substancialmente los niveles de apoyo emocional que tenían disponibles.

En resumen, la revisión de la literatura demostró tres razones para esperar más síntomas de depresión en las mujeres solteras con jefatura de familia. Primero, ser una mujer soltera con jefatura de familia es un estresor crónico, porque viola las normas de género tradicionales en la familia hispana. Segundo, este grupo tiene menos 
recursos que otras mujeres, ya que experimenta más pobreza y aislamiento. Aún más, el estrés continuo de ser mujer soltera con jefatura de familia, unido a otros estresores potenciales, como eventos de vida y responsabilidad hacia otras personas, podrían provocar más estrés en este grupo. Tercero, recursos como apoyo social, puede que no la protejan al nivel que ocurre con otras mujeres.

Se plantearon por tanto, las siguientes hipótesis: (1) Las mujeres solteras con jefatura de familia tienen más síntomas de depresión que otras mujeres. (2) Estas mujeres están más vulnerables a eventos estresantes que otras mujeres. (3) Las mujeres solteras con jefatura de familia no se benefician del apoyo social en la misma medida que otras mujeres.

Como se mencionó anteriormente, son escasos los estudios sobre la salud mental de mujeres solteras con jefatura de familia. Además los estudios previos, presentan diversas limitaciones. Es muy raro que enfoquen las mujeres solteras jefas de familia hispanas o las madres solteras hispanas (Belle, 1980; Bennett, 1987; McLanahan, 1985; Naran, 1989, y Wells y Ray, 1986). Aún más, los estudios que se han realizado en esta población están basados en muestras muy pequeñas (Colberg y Burgos, 1988; D'Ercole, 1988; Nuttall, 1979) o han incluido sólo mujeres mexicoamericanas (Vega et al., 1986 y 1987; Vernon, 1982). El presente estudio constituye un importante paso en la investigación transcultural sobre mujeres solteras con jefatura de familia ya que está basado en una muestra probabilística y contiene una serie de medidas de las circunstancias en la vida de las mujeres.

\section{METOdOLOGíA}

\section{Población y muestra}

La población en este estudio la componen mujeres entre las edades de 17 y 68 años residentes en Puerto Rico. Los datos analizados fueron recopilados en 1987 como parte de un estudio de epidemiología psiquiátrica realizado por el Instituto de Investigaciones del Recinto de Ciencias Médicas, con una muestra probabilística de 912 personas (523 mujeres y 388 hombres). La muestra incluyó tanto a las personas residentes en sus viviendas, como miembros de familias 
temporalmente fuera del hogar o aquellos en instituciones. Excluyó a los deambulantes o personas transitorias y aquellas que vivían en instituciones si su familia no residía en la comunidad. En el estudio se aumentó la probabilidad de incluir residentes que participaron en un estudio relacionado en el 1984. La tasa de respuesta de la encuesta fue de 91 por ciento. Detalles de la estrategia de muestreo han sido publicados previamente (Canino, et al., 1987a; Canino, et al., 1990).

El presente estudio tomó todas las mujeres de la muestra original que se encuentran en el banco de datos del Instituto de Investigaciones del Recinto de Ciencias Médicas. Para propósitos analíticos, las mujeres fueron clasificadas en tres grupos mutuamente exclusivos: mujeres solteras con jefatura de familia $(\mathrm{N}=138)$, mujeres solteras que no se denominaron a sí mismas como jefas de familia $(\mathrm{N}=104)$ y mujeres casadas quienes no eran jefas de familia $(\mathrm{N}=275)$. Había sólo seis mujeres casadas, que se clasificaban jefas de familia que fueron excluidas del estudio, para una muestra total de 517 mujeres.

\section{Procedimiento}

Los datos del estudio fueron recopilados en los hogares de los participantes con una planilla de entrevista estructurada. La misma fue administrada por diez entrevistadores(as) que recibieron adiestramiento en sesiones intensivas de cinco semanas y demostraron exitosamente su dominio de la planilla al entrevistar cuatro personas bajo estricta supervisión. Se utilizaron varias medidas para garantizar la calidad de los datos, las cuales tomaron en cuenta el muestreo, el trabajo de campo y el propio análisis de los datos como posibles fuentes de error, éstas fueron similares a las usadas en la encuesta previa (Vea Canino, et al., 1987b).

\section{Instrumentos}

\section{Síntomas de depresión}

Una versión en español reducida de la planilla de Entrevista Diagnóstica —en inglés: Diagnostic Interview Schedule (DIS) fue utilizada para ponderar los síntomas psiquiátricos. El DIS es una entrevista estructurada diseñada para ser utilizada por personas legas adiestradas. La misma produjo ponderaciones diagnósticas utilizando 
los criterios del DMS-III. Este instrumento fue traducido al español, adaptado y probado para su administración en la población puertorriqueña a través de un modelo transcultural abarcador que tomó en cuenta su equivalencia con la versión al inglés en varias dimensiones: semántica, de contenido, técnico, de criterio y conceptual (Bravo, et al., 1987; Bravo, et al., 1991). Su confiabilidad y concordancia con diagnósticos clínicos fueron similares a aquellos observados para la versión en inglés del instrumento (Canino, et al., 1987b; Robins, et al., 1981).

La versión reducida del DIS utilizada en este estudio cubre nueve categorías diagnósticas: episodios principales de depresión, distimia, desórdenes de estrés post-traumático, abuso y dependencia de alcohol y drogas, ansiedad generalizada, desorden de pánico y desórdenes de personalidad antisocial. Con el fin de incluir una variedad de síntomas de depresión, enfocamos en los síntomas asociados con un episodio principal de depresión o distimia más que en categorías diagnósticas. El desarrollo de una escala de síntomas de depresión utilizada ha sido explicada en otro artículo (Rubio, et al., 1989), donde se demostró su validez y confiabilidad (alpha=0.86). Para propósitos analíticos, los datos fueron transformados sacando la raíz cuadrada de la escala para normalizar la distribución de los residuales.

\section{Responsabilidad hacia otros}

Utilizamos tres medidas para ponderar el grado al cual las mujeres son responsables por otras personas. La primera es el número de hijos e hijas residiendo en el hogar. La segunda, es el número de hijos e hijas quienes viven en otro lugar y la tercera, es el número de familiares, como padres, madres o hermanos(as) quienes residen en su hogar.

\section{Apoyo social y estresores}

La planilla incluyó instrumentos para medir apoyo social y eventos de vida estresantes. El instrumento de apoyo social parte de la clasificación del apoyo en tres áreas: instrumental (provisión de bienes y servicios), la socio-emocional (cercanía e intimidad) y la 
recreativa (compañía en actividades sociales y recreativas). Para este estudio las variables de apoyo utilizadas incluyeron el número de personas mencionadas en la red de apoyo instrumental $($ alpha $=0.70)$ y emocional (alpha $=0.75){ }^{2}$ También se incluyeron otras dos variables de apoyo: la adecuacidad del apoyo (alpha=0.75) y la calidad del apoyo (alpha=0.66). La adecuacidad del apoyo se refiere a la percepción del sujeto de no necesitar más apoyo de personas o instituciones durante el pasado mes. La calidad del apoyo incluye el sentimiento de tener suficientes amigos(as), buenos o cercanos y de no sentirse sola (sentirse conectada a otros(as).

La sección de eventos de vida estresantes del instrumento usado en el estudio epidemiológico del Epidemiologic Catchment Area (ECA) fue utilizado para esta investigación (Regier, et al., 1984). Esta pregunta sobre la ocurrencia de eventos de vida en los últimos dos años. Los eventos incluidos en este estudio fueron: la pérdida de empleo o ingresos, terminación de la mejor amistad, asaltos, enfermedad, muerte de familiares o amigos(as). Se sumó el número de eventos que las mujeres informaron para obtener una medida de los eventos de vida estresantes.

\section{Variables de control}

Las variables de control usadas en el análisis fueron: edad medida en años, educación medida en años de escuela completados, ingreso medido en niveles de dólares y área de residencia codificada como una variable dicótoma, $(1=$ urbano y $0=$ rural $)$.

\section{Análisis}

Para el análisis presentado se utilizaron datos pesados que tomaron en consideración el esquema estratificado de muestreo usado en el estudio. Además, se incorporaron pesos adicionales para que la muestra se ajustara a la distribución por edad y género del censo de la población de 1980 .

\footnotetext{
${ }^{2}$ Nuestra medida de apoyo recreativo fue altamente correlacionada con la de apoyo emocional con un coeficiente de correlación Paerson de 0.6 ó mayor en los tres grupos. Por tal razón, excluimos el apoyo recreativo de nuestro análisis.
} 
Para ponderar diferencias entre los tres grupos de mujeres analizadas, utilizamos el procedimiento generalizado de modelo lineal (GLM en inglés) de SAS, utilizando variables predictorias continuas y categóricas. El primer segmento de análisis describe las diferencias entre los grupos de mujeres en todas las variables independientes y de control. El segmento de análisis que le sigue examina las diferencias en síntomas depresivos entre y dentro de los grupos. Estos análisis incluyeron examinar los efectos de interacción con el status marital y de jefatura de familia.

\section{HALLAZgos}

\section{Características socio-demográficas}

Las mujeres solteras con jefatura de familia eran relativamente mayores y menos educadas comparadas con los otros dos grupos (mujeres solteras sin jefatura y mujeres casadas). Los resultados en ingresos son congruentes con la literatura revisada, sobre los niveles de pobreza entre mujeres solteras con jefatura de familia. Éste fue el grupo más pobre, teniendo el ingreso anual familiar más bajo. A pesar de que la mayoría de las mujeres vivían en la zona urbana, el porcentaje de las mujeres solteras con jefatura de familia que vivían en esta zona fue significativamente más alto (Vea Tabla 1).

Tabla 1

Características sociodemográficas de las participantes

\begin{tabular}{lccrr}
\hline & $\begin{array}{c}\text { Mujeres } \\
\text { solteras con } \\
\text { jefatura de } \\
\text { familia }\end{array}$ & $\begin{array}{c}\text { Mujeres } \\
\text { solteras no } \\
\text { jefas de } \\
\text { familia }\end{array}$ & $\begin{array}{c}\text { Mujeres } \\
\text { casadas }\end{array}$ & F- valor \\
\hline Edad & 45.7 & 29.2 & 42.0 & $50.5^{* * *}$ \\
Educación & 8.8 & 13.4 & 10.8 & $28.3^{* * *}$ \\
$\begin{array}{l}\text { Ingreso } \\
\text { Area de residencia }\end{array}$ & $\$ 7,997$ & $\$ 12,440$ & $\$ 14,201$ & $9.0^{* * *}$ \\
$\%$ urbano & 83.3 & 66.0 & 59.8 & $8.0^{* *}$ \\
\hline Nota: ${ }^{* *} \mathrm{p}<.01$ & & & & \\
${ }^{* * *} \mathrm{p}<.001$ & & & & \\
\end{tabular}


En relación a las circunstancias estresantes, los grupos fueron diferentes en todas las medidas de responsabilidad hacia otros y en los eventos de vida (Vea Tabla 2). Las mujeres solteras jefas de familia tenían menos hijos(as) en el hogar que las casadas, pero tenían significativamente más hijos en el hogar que las solteras que no eran jefes de familia. Éstas también tenían más hijos(as) fuera del hogar que las otras mujeres y tendían a vivir menos con otros familiares. En promedio, las mujeres solteras con jefatura de familia eran más propensas a haber pasado por uno o más eventos de vida estresantes que las casadas. Sin embargo, estos dos grupos, eran menos propensos a pasar por estos eventos que las solteras no jefas de familia.

Tabla 2

Estresores: Responsabilidad por otros y eventos de vida estresantes

\begin{tabular}{lcccc}
\hline & $\begin{array}{c}\text { Mujeres } \\
\text { solteras con } \\
\text { jefatura de } \\
\text { familia }\end{array}$ & $\begin{array}{c}\text { Mujeres } \\
\text { solteras no } \\
\text { jefas de } \\
\text { familia }\end{array}$ & $\begin{array}{c}\text { Mujeres } \\
\text { casadas }\end{array}$ & F - valor \\
\hline $\begin{array}{l}\text { Responsabilidad por otros } \\
\text { Hijos(as) en el hogar }\end{array}$ & 1.5 & 0.3 & 1.7 & $50.2^{* * *}$ \\
Hijos(as) en el hogar & 1.6 & 0.1 & 1.4 & $21.1^{* * *}$ \\
$\begin{array}{l}\text { Parientes en el hogar } \\
\text { Eventos de vida estresantes }\end{array}$ & 0.8 & 2.8 & 1.2 & $134.6^{* * *}$ \\
$\%$ informando uno o más & 25.5 & 43.1 & 25.8 & $4.9^{* *}$ \\
\hline Nota: ** $\mathrm{p}<.01$ & & & & \\
\hline
\end{tabular}

La Tabla 3 demuestra que, con la excepción de la calidad del apoyo, los grupos no difieren en medidas de redes de apoyo social. Las entrevistadas tendían a informar apoyos instrumentales y sociales similares, y a caracterizar el apoyo como adecuado. No obstante, los dos grupos de mujeres solteras informaron niveles de satisfacción con el apoyo más bajos que las casadas. Por lo tanto, sin importar el tamaño de su red de apoyo emocional e instrumental, ni la percepción de adecuacidad del apoyo recibido, las mujeres solteras tendían a sentirse más aisladas y sin suficientes amistades cercanas (indicadores de la satisfacción con el apoyo). 
Tabla 3

Apoyo Social

\begin{tabular}{lcccc}
\hline & $\begin{array}{c}\text { Mujeres } \\
\text { solteras con } \\
\text { jefatura de } \\
\text { familia }\end{array}$ & $\begin{array}{c}\text { Mujeres } \\
\text { solteras no } \\
\text { jefas de } \\
\text { familia }\end{array}$ & $\begin{array}{c}\text { Mujeres } \\
\text { casadas }\end{array}$ & F - valor \\
\hline Número en instrumental & 3.5 & 4.0 & 3.8 & 1.6 \\
Número en emocional & 3.6 & 3.9 & 4.0 & 1.8 \\
Calidad del apoyo & 6.7 & 6.8 & 7.3 & $8.0^{* * *}$ \\
Adecuacidad & 1.6 & 1.6 & 1.7 & 1.3 \\
\hline
\end{tabular}

Nota: ${ }^{* * *} \mathrm{p}<.001$

\section{Síntomas de depresión}

La siguiente sección de análisis se enfoca en los niveles de los síntomas de depresión. Como se esperaba, los grupos tuvieron diferentes niveles de síntomas de depresión, las mujeres solteras con jefatura de familia mostraron niveles promedio más altos (1.34) que las solteras no jefas de familia (1.14), y las casadas (0.99). Habíamos hipotetizado que las mujeres solteras con jefatura de familia tendrían niveles más altos de depresión por la limitación de recursos económicos y sociales y porque se desviaban de las normas de género. Ya que no teníamos un indicador directo que midiera normas de género no pudimos examinar directamente esa hipótesis. Sin embargo, aparentemente las diferencias en recursos como el ingreso y la educación no explicaron los niveles altos de síntomas de depresión de las mujeres solteras con jefatura de familia; cuando controlamos estadísticamente para área de residencia, ingreso y educación, aún persistieron los niveles más altos de síntomas en las solteras jefas de familia (Vea Tabla 4).

Con el objetivo de examinar, no sólo si los niveles de síntomas variaban entre los tres grupos de mujeres, sino también la naturaleza de los mismos, comparamos la frecuencia y el rango de los síntomas de las mujeres solteras con jefatura de familia con los de las solteras sin jefatura y las mujeres casadas (Vea Tabla 5).

Las mujeres solteras con jefatura de familia y las mujeres casadas mostraron un perfil muy similar de síntomas (coeficiente de 
Tabla 4

Nivel de Síntomas de Depresión

\begin{tabular}{lcccc}
\hline & $\begin{array}{c}\text { Mujeres } \\
\text { solteras con } \\
\text { jefatura de } \\
\text { familia }\end{array}$ & $\begin{array}{c}\text { Mujeres } \\
\text { solteras no } \\
\text { jefas de } \\
\text { familia }\end{array}$ & $\begin{array}{c}\text { Mujeres } \\
\text { casadas }\end{array}$ & F - valor \\
\hline $\begin{array}{l}\text { Promedio de } \\
\text { síntomas depresivos: }\end{array}$ & 1.34 & 1.14 & 0.99 & $5.3^{* *}$ \\
$\begin{array}{l}\text { Sin ajustar } \\
\text { Ajustado }\end{array}$ & 1.33 & 1.11 & 1.10 & $5.1^{* *}$ \\
\hline Nota: ${ }^{\text {a }}$ Ajustado por área de residencia, ingreso, educación, y edad. & \\
${ }^{* *}$ p $<.01$ & & & &
\end{tabular}

correlación de rango $=.90$ ), aun cuando difirieron en el nivel de síntomas, como se indicó antes. Sin embargo, el perfil de las mujeres solteras con y sin jefatura de familia fue algo distinto (coeficiente de correlación de rango $=.72$ ). Las diferencias principales entre estas últimas ocurrió en los síntomas referentes a "desear morirse" y "dificultad en concentrarse". Mientras que para las mujeres solteras con jefatura de familia el "desear morirse" fue el $9^{\circ}$ ítem más frecuente, para las mujeres solteras sin jefatura de familia fue el $15^{\circ}$; un patrón totalmente contrario ocurrió con la "dificultad para concentrarse" $\left(9^{\circ}\right.$ para las sin jefatura y $15^{\circ}$ para las con jefatura). También difirieron estos dos grupos en el síntoma básico del trastorno de distimia, "ánimo deprimido la mayor parte del tiempo", que fue el $7^{\circ}$ más frecuente para las solteras con jefatura pero el $12^{\circ}$ para aquellas que no tienen jefatura.

FACTORES PSICOSOCIALES. En este segmento de análisis también se examinó las asociaciones de los factores psicosociales y los síntomas de depresión. Para determinar si estas asociaciones diferían por status de jefatura, probamos para los efectos de interacción. Debido a que varias de estas interacciones fueron estadísticamente significativas, presentamos resultados de regresión separados para cada uno de los grupos. Éstos aparecen en la Tabla 6, y demuestran las asociaciones de los síntomas de depresión con medidas de responsabilidad, eventos de vida y apoyo social, después de controlar por variables sociodemográficas. 
Tabla 5

Perfil de síntomas de depresión

\begin{tabular}{|c|c|c|c|c|c|c|}
\hline \multirow{2}{*}{$\begin{array}{l}\text { Descripción del síntoma } \\
\text { Muchos ataques de llantob }\end{array}$} & \multicolumn{2}{|c|}{$\begin{array}{l}\text { MSJFa } \\
\text { Frec. } \\
\text { (rango) }\end{array}$} & \multicolumn{2}{|c|}{$\begin{array}{l}\text { MSSJF } \\
\text { Frec. } \\
\text { (rango) }\end{array}$} & \multicolumn{2}{|c|}{$\begin{array}{c}\text { MC } \\
\text { Frec. } \\
\text { (rango) }\end{array}$} \\
\hline & 39 & (3) & 23 & (3) & 81 & (1) \\
\hline Período de desesperanza & 49 & (2) & 32 & (1.5) & 65 & (3) \\
\hline $\begin{array}{l}\text { Ánimo consistentemente } \\
\text { muy deprimido }\end{array}$ & 66 & (1) & 32 & (1.5) & 79 & (2) \\
\hline $\begin{array}{l}\text { Ánimo deprimido la mayor } \\
\text { parte del tiempo }\end{array}$ & 24 & (7) & 8 & (12.5) & 34 & $(5.5)$ \\
\hline Pérdida de apetito & 16 & $(10)$ & 16 & (6) & 28 & (7) \\
\hline Pérdida involuntaria de peso & 21 & $(8)$ & 19 & (5) & 21 & (10.5) \\
\hline Problemas con el sueño & 34 & (4) & 20 & (4) & 48 & (4) \\
\hline $\begin{array}{l}\text { Agotamiento o mucho } \\
\text { cansancio }\end{array}$ & 25 & (6) & 14 & (7.5) & 24 & (8) \\
\hline Continuo movimiento & 13 & $(12.5)$ & 8 & (12.5) & 19 & (12.5) \\
\hline Sentimiento de minusvalía & 6 & (16) & 8 & (12.5) & 8 & (16) \\
\hline Dificultad en concentrarse & 8 & (15) & 13 & (9) & 21 & (10.5) \\
\hline $\begin{array}{l}\text { Pensamientos lentos o } \\
\text { confusos }\end{array}$ & 14 & (11) & 5 & (16) & 23 & (9) \\
\hline Pensar en la muerte & 29 & (5) & 14 & (7.5) & 34 & (5.5) \\
\hline Desear morirse & 20 & (9) & 7 & (15) & 19 & (12.5) \\
\hline Pensar en suicidarse & 13 & (12.5) & 8 & (12.5) & 17 & (14) \\
\hline Intentar suicidarse & 11 & (14) & 9 & (10) & 11 & (15) \\
\hline
\end{tabular}

a $M S J F=$ Mujeres solteras jefas de familia; MSSJF $=$ Mujeres solteras $\sin$ jefatura de familia; $\mathrm{MC}=$ Mujeres casadas.

b Los períodos de tiempo respecto a los que inquieren los ítemes son a lo largo de la vida; para ánimo deprimido la mayor parte del tiempo el período es de dos años; los demás, dos semanas

RESPONSABILIDAD POR OTROS. Esperábamos que el tener hijos(as) o dependientes sería más estresante para las mujeres solteras con jefatura de familia que para las otras mujeres. Nuestra hipótesis fue confirmada sólo con respecto a los hijos(as) en el hogar. La interacción entre esta variable y la de estado marital/jefatura fue estadísticamente significativa $(\mathrm{F}=5.52, \mathrm{p}<0.005)$. Al analizar los resultados un 
Tabla 6

Regresión de síntomas depresivos en variables sociodemográficas, responsabilidad por otros, estrés, y apoyo social

\begin{tabular}{|c|c|c|c|c|c|c|}
\hline \multirow[b]{2}{*}{ Área de residencia } & \multicolumn{2}{|c|}{$\begin{array}{r}\text { MSJF } \\
\text { b (s.e.) }\end{array}$} & \multicolumn{2}{|c|}{$\begin{array}{l}\text { MSNJF } \\
\text { b (s.e.) }\end{array}$} & \multicolumn{2}{|c|}{$\begin{array}{c}\text { MC } \\
\text { b (s.e.) }\end{array}$} \\
\hline & .484 & $(.197)$ & .270 & $(.211)$ & .144 & $(.128)$ \\
\hline Ingreso & .003 & $(.011)$ & .045 & $(.010)$ & .033 & $(.004)$ \\
\hline Educación & -.020 & $(.018)$ & -.040 & $(.038)$ & $-.046^{\star \star}$ & $(.016)$ \\
\hline Edad & -.009 & $(.007)$ & $.034^{\star \star}$ & $(.010)$ & .016 & $(.006)$ \\
\hline Hijos(as) en el hogara & $.192^{\star \star \star}$ & $(.053)$ & $.943^{\star \star}$ & $(.255)$ & .027 & $(.045)$ \\
\hline Hijos(as) fuera del hogar & .039 & $(.039)$ & .143 & $(.389)$ & -.051 & $(.037)$ \\
\hline Parientes en el hogar & .047 & $(.065)$ & -.097 & $(.068)$ & $-.198^{\star}$ & $(.100)$ \\
\hline $\begin{array}{l}\text { Eventos de vida } \\
\text { estresantes }\end{array}$ & .033 & $(.154)$ & .036 & $(.048)$ & .036 & $(.111)$ \\
\hline $\begin{array}{l}\text { Número de apoyadores } \\
\text { instrumental }\end{array}$ & .028 & $(.037)$ & .045 & $(.047)$ & .031 & $(.028)$ \\
\hline $\begin{array}{l}\text { Número de apoyadores } \\
\text { emocional }^{\mathrm{a}}\end{array}$ & $.061^{+}$ & $(.037)$ & -.069 & $(.051)$ & $-.071^{\star *}$ & $(.026)$ \\
\hline Adecuacidad & $-.402^{\star \star \star}$ & $(.113)$ & -.230 & $(.129)$ & $-.243^{\star \star \star}$ & $(.100)$ \\
\hline Calidad del apoyo ${ }^{a}$ & $-.219^{\star \star \star}$ & $(.045)$ & -.018 & $(.071)$ & $-.333^{\star \star \star}$ & $(.047)$ \\
\hline $\mathrm{R}^{2}$ & 0.3 & 39 & 0.3 & & 0. & 32 \\
\hline
\end{tabular}

Nota: a Interacción con status de jefatura significativo en $\mathrm{p}<.05$

$+\mathrm{p}<.10$

${ }^{*} \mathrm{p}<.05$

** $\mathrm{p}<.01$

*** $\quad \mathrm{p}<.001$

poco más (Tabla 6), encontramos que la presencia de hijos(as) estaba positivamente asociada con síntomas para los dos grupos de mujeres solteras, pero no así para las mujeres casadas.

EVENTOS DE VIDA ESTRESANTES. Cuando examinamos la contribución de los eventos de vida estresantes, encontramos que el número de eventos estaba marginalmente asociado con los síntomas en todas las mujeres $(\mathrm{b}=0.14, \mathrm{t}=1.63, \mathrm{p}<0.10) .{ }^{3}$ No obstante, la

${ }^{3}$ El añadir las medidas de apoyo social a la ecuación reduce este coeficiente a no significativo, indicando que el apoyo es mediador del efecto de los eventos de vida estresantes para esta población. 
interacción de eventos de vida y la variable de estado marital/jefatura no fue significativa, sugiriendo que el impacto de estos estresores no varía en los tres grupos de mujeres.

APOYO SOCIAL. Encontramos interacciones significativas con la variable de estado marital/jefatura en dos de las cuatro medidas de apoyo: número de personas en la red de apoyo emocional y satisfacción con el apoyo. Cuando miramos los grupos en forma separada (Vea Tabla 6), encontramos que el efecto de apoyo emocional fue significativo sólo para mujeres casadas, a pesar de que el coeficiente de apoyo emocional fue también negativo y similar en magnitud para las mujeres solteras no jefas de familia. Aún más, el apoyo emocional estuvo relacionado positivamente a los síntomas de las mujeres solteras con jefatura de familia, pero esta relación fue significativa solamente a un nivel marginal $(p<.10)$. La disponibilidad del apoyo instrumental no estuvo relacionado a los síntomas en ninguno de los grupos.

La Tabla 6 también demuestra que la calidad del apoyo (conexión social a otras personas) estuvo negativamente asociada con síntomas tanto para las mujeres solteras con jefatura de familia como para las casadas; pero no hubo asociación para las solteras no jefas de familia. A pesar de que la Tabla 6 también sugiere que la adecuacidad del apoyo no estuvo significativamente relacionada con síntomas entre las mujeres solteras no jefas de familia, la prueba de interacciones indicó que el coeficiente de adecuacidad para este grupo $(-0.23)$ no difería significativamente del de los otros grupos. En otras palabras, la adecuacidad estuvo significativa y negativamente relacionada con los síntomas para las casadas y solteras jefas de familia.

En resumen, observamos que las mujeres solteras con jefatura de familia mostraron un mayor nivel de síntomas de depresión que las otras aun cuando se controlara por ingreso de educación y área de residencia. Sin embargo, su perfil de síntomas sólo fue distinto que el de las mujeres solteras no jefas de famila. La presencia de hijos en el hogar se encontró que estuvo positivamente asociada con síntomas en los dos grupos de mujeres solteras, pero no en las casadas. Las mujeres solteras jefas de familia no manifestaron menos síntomas de depresión al tener más apoyo emocional disponible, contrario 
a los otros dos grupos de mujeres en que el apoyo sí tuvo este efecto. Las mujeres solteras con jefatura de familia se visualizan como menos conectadas socialmente a otras personas, pero cuando se sienten más conectadas muestran menos síntomas.

\section{Discusıón}

Nuestro hallazgo de que las mujeres solteras con jefatura de familia son especialmente más vulnerables a síntomas de depresión es congruente con los resultados de otros estudios (Belle, 1980; Morrison et al., 1986; Vega et al., 1986; D'Ercole, 1988). Al igual que en investigaciones previas, cuando se compara a las mujeres solteras con jefatura de familia con otras mujeres, éstas eran mayores, más pobres, con menos educación y residían en mayor número en zonas urbanas. Sin embargo, las variaciones en estas características no explicaron las diferencias en sintomatología depresiva, ya que las mujeres solteras con jefatura de familia exhibieron mayores niveles de síntomas que las casadas y solteras no jefas de familia, después de controlar los factores socio-demográficos.

Examinamos también variables psicosociales para identificar circunstancias que podrían explicar la alta prevalencia de síntomas entre las mujeres solteras con jefatura de familia. Hipotetizamos que gran parte del estrés de ser soltera con jefatura de familia se debía a la responsabilidad de tener hijos(as) dentro y fuera de la casa, como también el tener dependientes en el hogar. Confirmamos parcialmente esta hipótesis. Como se esperaba, las mujeres solteras exhibieron mayores niveles de síntomas cuando tenían hijos(as) en el hogar, pero, contrario a nuestras expectativas, ser jefa de familia no añadía a esta vulnerabilidad ya que este patrón fue también observado para solteras no jefas de familia. La falta de un compañero estable con quien compartir las demandas que esta responsabilidad impone es probablemente un factor más importante para estas mujeres solteras. Contrario a nuestras expectativas, las mujeres con hijos(as) fuera del hogar o parientes dependientes en el hogar demostraron niveles similares de síntomas sin importar el estado marital o de jefatura. Es decir, este tipo de responsabilidad evoca un efecto similar en los tres grupos de mujeres. 
El examen de la naturaleza de los síntomas demostró unas diferencias entre las mujeres solteras con y sin jefatura de familia. Tal fue el caso para los síntomas de depresión "desear morirse" y "ánimo deprimido la mayor parte del tiempo por dos (2) años" que ocurrió más en las mujeres solteras con jefatura de familia que en las solteras que no eran jefas. Este dato sugiere que las diferencias en el perfil de síntomas están asociadas a la vulnerabilidad debida al desempeño de la jefatura de familia entre las mujeres solteras.

Exploramos también varios factores que podrían aumentar el riesgo de síntomas depresivos entre las mujeres solteras con jefatura de familia. Después de tomar en consideración los factores sociodemográficos, examinamos el estrés y el apoyo social. Se encontró que los eventos estresantes no explicaban las diferencias entre las mujeres solteras con jefatura de familia y las otras mujeres. Este resultado no es congruente con estudios previos que han encontrado a las mujeres solteras en más alto riesgo de angustia psicológica luego de eventos estresantes (Thoits, 1987). Esta discrepancia puede ser debido a diferencias en las muestras estudiadas. Este asunto merece ser estudiado en investigaciones futuras con otros grupos de mujeres hispanas para ver si se aplica nuestro hallazgo.

Al considerar el rol del apoyo social, identificamos aspectos del apoyo que afectaban a los distintos grupos de mujeres en forma diferente. Las mujeres solteras no jefas de familia y las casadas exhibieron niveles más bajos de síntomas depresivos cuando tenían más apoyo emocional disponible. Sin embargo, ése no fue el caso para las mujeres solteras con jefatura de familia; la disponibilidad de apoyo emocional no las beneficiaba de la misma manera que a las otras mujeres. De hecho, se observó un efecto marginal positivo y significativo del apoyo emocional en los síntomas para las mujeres solteras con jefatura de familia. Este resultado es congruente con nuestra hipótesis de que el apoyo social no beneficia a estas mujeres en la medida que beneficia a las demás. Una posible explicación puede encontrarse en la presión sutil impuesta por la cultura puertorriqueña a las mujeres solteras con jefatura de familia para que tengan o busquen una pareja permanente para que asuma la jefatura de familia. Aún más, las mujeres pueden tener un buen número de personas que brinden apoyo emocional pero puede ser que estas 
personas no sean capaces de satisfacer todas las necesidades emocionales, especialmente aquellas que supuestamente satisface un esposo o compañero. Futuras investigaciones podrían comparar la necesidad y las fuentes de apoyo emocional de las mujeres solteras con jefatura de familia y otras mujeres. Además, estas investigaciones pueden considerar el rol del género en la determinación de la adecuacidad del apoyo.

Las mujeres solteras, jefas y no jefas de familia, se visualizan ellas mismas como menos conectadas socialmente a otras personas que las casadas. No obstante, para las mujeres solteras jefas de familia como también para las casadas, el sentirse conectadas a otras personas se asocia con menos sintomatología. Es decir, a pesar de que las mujeres solteras con jefatura de familia se sienten menos conectadas, cuando ellas se perciben como tal, se benefician de esta experiencia. Este resultado sugiere que la calidad del apoyo es más importante para las solteras con jefatura de familia que el número de personas disponibles para proveer apoyo emocional.

Los resultados de este estudio tienen que entenderse a la luz de la naturaleza transversal de la muestra, lo cual hace problemático las inferencias de causalidad. Por ejemplo, es posible que las asociaciones entre apoyo social y síntomas de depresión surjan porque la mujer depresiva se sienta desconectada de otras personas o porque se perciba como que no están recibiendo el apoyo emocional adecuado. A pesar de que esas interpretaciones son posibles, no explican todos los hallazgos, como es el efecto diferencial del apoyo emocional para las mujeres solteras con jefatura de familia comparadas con otras mujeres. Sin embargo, solamente la investigación prospectiva puede clarificar estos aspectos de causalidad. Además, se tiene que considerar la limitación que plantea un banco de datos que se ha diseñado con otros propósitos como ha sido el utilizado para este análisis. Estudios especialmente diseñados para estudiar el tema que aquí nos ocupa, podrían incluir otras variables pertinentes para entender más a cabalidad la situación de las mujeres solteras jefas de familia. 


\section{IMPLICACIONES PARA LAS PROFESIONES DE AYUDA}

No cabe duda que estamos ante un segmento de la población que está en aumento y es muy vulnerable. Es necesario estimular a estas mujeres a que mejoren la calidad de su situación. Uno de los medios que pueden usar para lograr esto, es a través de sus redes de apoyo. Los grupos de discusión pueden ser una alternativa a ser considerada, para mejorar la calidad de estas redes, ya sea utilizando a grupos ya existentes o formando grupos de mujeres solteras con jefatura de familia. Es recomendable que este grupo logre lidiar con el conflicto de roles, entendiendo el proceso de formación de género en esta cultura, cómo los roles instrumental y expresivo pueden ser ejercidos por ambos géneros y que no tienen que ser mutuamente exclusivos. Probablemente tengamos que lidiar con la angustia y ansiedad de las mujeres que se sienten solas porque no tienen marido o compañero permanente, especialmente cuando tienen hijos en la casa. Se puede fortalecer a la mujer ayudándola a que se sienta bien con ella misma y darle énfasis a que ella no está sola, pues tiene a su familia y sus amistades.

En Puerto Rico se ha acumulado una experiencia de cuatro años con grupos de apoyo y discusión para mujeres con jefatura de familia en el Centro Universitario de Servicios y Estudios Psicológicos (CUSEP). Estos grupos han provisto un espacio para que las mujeres puedan compartir sus situaciones en la estructura constituida por ellas; han ofrecido una alternativa de cambio futura, al iniciar estructuras de ideologías que consideran las experiencias de las mujeres y una alternativa inmediata de un grupo de discusión de estas vivencias que a la vez le brinda apoyo social y psicológico (Colberg, 1995).

Un aspecto educativo, que con frecuencia resulta necesario, es el desarrollo de destrezas para el trabajo, especialmente si han estado fuera de la fuerza laboral por mucho tiempo. Muchas también, necesitan toda una preparación para el mundo del trabajo desde lo más simple hasta lo más complejo. Las mujeres que opten por permanecer en el hogar cuidando a sus hijos, probablemente necesitan orientación legal y sobre los programas gubernamentales de asistencia económica. Asimismo, las que deciden estudiar requieren de una prepa- 
ración para tales fines, especialmente si han estado fuera de la escuela o si deciden comenzar una carrera técnica o universitaria. Con las altas tasas de divorcio en Puerto Rico, es posible que este sector del grupo de mujeres solteras con jefatura de familia, necesite más atención. No obstante, aquí nos puede ayudar la literatura existente de intervención con divorciadas(os), como por ejemplo los trabajos de Muñoz y Fernández (1988).

Es necesario, también explorar otras necesidades de las mujeres solteras con jefatura de familia. Por ejemplo: la necesidad de diversos tipo de cuidado infantil y atención a menores. El cuidado infantil no debe verse solamente como una necesidad de las madres que trabajan fuera del hogar, sino también como un servicio a madres que tienen que resolver todos los problemas que atañen a la familia bajo su responsabilidad. Además, las actividades después de la escuela, ya sean de tutoría o de recreación, pueden resultar apropiadas para niños y niñas en edad escolar. A nivel nacional tenemos que reconocer que la mujer soltera con jefatura de familia es sostenedora de un tipo de familia, que estas familias no deben evaluarse como incompletas y menos, desviadas. Desde los grados primarios se debe hablar de los diferentes tipos de familia, incluyendo estas familias, para que los niños(as) de las mismas no se sientan diferentes y no se siga perpetuando el estigma social.

\section{Reconocimientos}

Esta investigación pudo ser realizada con fondos del Instituto Nacional de Salud Mental (Grant R01 MH48894). Las autoras desean reconocer la participación en la investigación, como estadísticos, de los doctores Paul Clearly y Josué Guzmán; a la analista de datos Zenaida González y a Migdalia Alejandro por el trabajo mecanográfico. Un agradecimiento especial a la Dra. Glorisa Canino y a Maritza Rubio por el asesoramiento en la elaboración de la propuesta y por el acceso a su banco de datos.

\section{Resumen}

Este artículo está basado en una investigación sobre el riesgo de síntomas de depresión en mujeres solteras con jefatura de familia y 
cómo los sistemas de apoyo no parecen beneficiarles, en comparación con otras mujeres en Puerto Rico. El análisis está basado en un banco de datos sobre una muestra probabilística de la población adulta de 17 a 68 años de toda la Isla. Las mujeres solteras con jefatura son un segmento de la población en crecimiento, que resultaron ser más pobres, con menor educación, más viejas y concentrado en zonas urbanas. Se concluye con algunas implicaciones para las profesiones de ayuda.

\section{Referencias}

Barrera, M., Jr. (1986). Distinctions between social support concepts, measures and models. American Journal of Community Psychology, 14(4), 413-445.

Belle, D. (1980). Stress and depression: symptoms of the times. Innovations, 7(3), 48.

Bem, S.L. (1974). The measurement of psychological androgyny.Journal of Consulting and Clinical Psychology, 42, 155-162.

Bennett, M.B. (1987). Afro-American women poverty and mental health: a social essay: Women and Health, $12(3-4), 213-228$.

Bravo, M.; Canino, G. y Bird H. (1987). El DIS en español: Su traducción y adaptación en Puerto Rico. Acta Psiquiátrica y Psicológica de América Latina, 33, 27-42.

Bravo, M.; Canino, G.; Rubio-Stipec, M. y Woodbury-Fariña, M. (1991). A crosscultural adaptation of a psychiatric epidemiologic instrument: The diagnostic interview schedule's adaptation in Puerto Rico.Culture, Medicine and Psychiatry, $15,1-18$

Brown, G.W. y Harris, T. (1978). Social origins of depression, New York: Free Press.

Burgos, N.M. (1986). Women, work and family in Puerto Rico. AFFILIA-Journal of Women and Social Work, 1 (3), 17-28, (Fall).

Canino, G.; Rubio-Stipec, M.; Shrout, P.; Bravo, M.; Stolberg, R. y Bird, H.R. (1987a). Sex differences and depression in Puerto Rico. Psychology of Women Quarterly, 11, 443-459.

Canino, G.; Bird, H.; Shrout, P.E.; Rubio-Stipec, M.; Bravo, M.; Martínez, R.; Sesman, M. y Guevara, L.M. (1987b). The prevalence of specific psychiatric disorders in Puerto Rico. Archives of General Psychiatry, 44, 727-735 (August).

Canino, G.; Bravo, M.; Rubio-Stipec, M. y Woodbury, M. (1990). The impact of disaster on mental health: Prospective and retrospective analysis. International Journal of Mental Health, 19(1), 55-69. 
Cohen, S.; Syme, L. (1985). Issues in the study and applications of social support. En S. Cohen y L. Syme (Eds.), Social support and health (pp. 3-22). Orlando, FL: Academic Press.

Colberg, E.M. (1995). Manual de grupos de discusión y apoyo de mujeres. Sometido a publicación al Centro de Investigaciones Sociales, Universidad de Puerto Rico, Río Piedras.

Colberg, E.M. y Burgos, N.M. (1988). Female-headed single parent families in Puerto Rico: an exploratory study of work and family conditions. Journal of Social Behavior and Personality, 3(2), 373-387.

Costin, L.B. (1987). Is the family neglectful or neglected? Society, 24, 3(167), 27-32 (mar-abr.).

D'Ercole, A. (1988). Single mothers: stress, coping, and social support. Special issue: Women in the community. Journal of Community Psychology, 16(1), 41-54. (Ene.).

Dohrenwend, B.S. y Dohrenwend, B.P. (1981). Life stress and illness: Formulation of the issues. En B.S. Dohrenwend y B.P. Dohrenwend (Eds.), Stressful life events and their contexts, (pp. 1-27). New York: Prodists.

Holmes, T.H. y Rahe, R.H. (1967). The social readjustment rating scale. Journal of Psychosomatic Research, 11, 213-218.

House, J.S. (1981). Work stress and social support. Reading, MA: Addison-Wesley.

Kamerman, S.B. y Kahn, A.J. (1988). Mothers alone: Strategies for a time of change. Massachusetts: Auburn House Publishing, Co.

Lipman-Blumen, J. (1984). Gender, roles and power. New Jersey: Prentice-Hall, Inc.

McLanaham, S. (1985). Single mothers and psychological well-being: A test of the stress and vulnerability hypothesis. Research in Community and Mental Health, 5, 253-266.

Morrison, W., et al. (1986). Single mothers in Canada: An analysis. Canadian Journal of Community Mental Health, 5(2), 37-47. (Otoño).

Muñoz, M. y Fernández, E. (1988). El divorcio en la sociedad puertorriqueña. Río Piedras, Puerto Rico: Ediciones Huracán.

Myers, J.K; Weissman, M.M.; Tischler, G.L. et al. (1984). Six month prevalence of psychiatric disorders in three communities. Archives of General Psychiatry, 10, 959-970.

Naran, R. (1989). Women and their social networks: A focus on single mothers. Doctoral Dissertation, University of California at Berkeley.

Nuttall, E.V. (1979). The support system and coping patterns of the female Puerto Rican single parent. Journal of Non-White Concerns in Personnel and Guidance, 7(3), 126-137. (Abril).

Regier, D.A.; Myers, J.K.; Dramer, M.; Robins, L.N.; Balzer, D.G.; Hough, R.L.; Eaton, W.W. y Locke, B.Z. (1984). The NIMH epidemiologic catchment area program. Archives of General Psychiatry, 40, 1183-1188. 
Robins, L.N.; Helzer, J.E.; Weissman, M.M.; Orvaschel, H.; Gruenberg, E.; Burke, J.D. y Regier, D.A. (1984). Lifetime prevalence of specific psychiatric disorders in three sites. Archives of General Psychiatry, 10, 949-958.

Rubio-Stipec, M.; Shrout, P.; Bird, H.; Canino, G. y Bravo, M. (1989). Symptom scales of the diagnostic interview schedule factor results in hispanic and anglo samples. Journal of Consulting and Clinical Psychology. 1, 30-34.

Sánchez, C.D. (1989). Informal support systems of widows over 60 in Puerto Rico. En American Association of Retired Persons, (Eds.), Mid-life and older women. Washington, D.C.: Pan American Health Organization.

Solomon, S.D.; Bravo, M.; Rubio-Stipec, M. y Canino, G. (1993). Effect of family role on response to disaster. Journal of Traumatic Stress, 6(2), 255-269.

Tardy, C.H. (1985) Social support measurement. American Journal of Community Psychology, 13 (2), 187-202.

Thoits, P.A. (1987). Gender and marital status differences in control and distress: Common stress versus unique stress explanations. Journal of Health and Social Behavior, 28, 7-22.

U.S. Bureau of the Census (1991a). Census of population: General population characteristics. Washington: U.S. Government Printing Office.

U.S. Bureau of the Census (1991b). Census of Population and Housing: Puerto Rico. Washington: U.S. Government Printing Office.

U.S. Bureau of the Census (1972). General Social and Economic Characteristic: Puerto Rico. Washington, DC: US Government Printing Office.

Vega, W.A. et al. (1986). The relationship of marital status, confidant support and depression among Mexican immigrant women. Journal of Marriage and the Family, 48, 597-605.

Vega, W.A.; Kolody, B. y Valle, J.R. (1987). Migration and mental health: an empirical test of depression risk factors among immigrant Mexican women. International Migration Review, 21, 512-530.

Vernon, S.W. y Roberts, R.E. (1982). Use of the SADS-RDC in a triethnic community survey. Archives of General Psychiatry, 39, 47-52.

Weissman, M.M. y Klerman, G.L. (1978). Epidemiology of mental disorder: Emerging trends in the U.S. Arch Gen Psychiatry, 35, 705-712.

Wells Gladow, N. y Ray Margaret, P. (1986). The impact of informal support systems on the well being of low income single parents. Family Relations, 35(1)m, 113 123.

Zegans, L.S. (1985). Stress and the development of somatic disorders. En Goldberg, L. y Breznitz, S. (Eds.), Handbook of Stress: Theoretical and Clinical Aspects, (pp. 134-152). New York: Free Press. 